\title{
Methyl group dynamics in glassy systems: Crossover from quantum to classical regime
}

\author{
A. J. Moreno, A. Alegría, and J. Colmenero \\ Departamento de Física de Materiales y Centro Mixto CSIC-UPV/EHU, Universidad del País Vasco (UPV/EHU), Apartado 1072, \\ 20080 San Sebastián, Spain
}

(Received 27 October 2000; published 3 January 2001)

\begin{abstract}
The problem of the crossover from quantum to classical dynamics for methyl group rotation in glassy systems is tackled in a quantitative way. A barrier distribution model, which was previously introduced to explain the neutron-scattering spectra in the low- and high-temperature limits, is extended now to the whole temperature range without introducing additional fitting parameters and it is checked in poly(vinyl acetate) with excellent results.
\end{abstract}

\section{DOI: 10.1103/PhysRevB.63.060201}

The crossover from coherent quantum tunneling to relaxation is a particular case of the general and fascinating problem of the transition from quantum to classical dynamics. Nowadays it is well accepted that this transition occurs due to the loss of quantum coherence ("decoherence") which emerges as a consequence of the coupling of the system to the many coordinates characterizing the environment. ${ }^{1}$ Small molecular rotors as the symmetric methyl groups $\left(-\mathrm{CH}_{3}\right)$, which only have one rotational degree of freedom, have been considered as ideal systems for monitoring and understanding this transition [see, e.g., (Refs. 2-5)]. In this case, the "environment", is given by the phonons of the matrix (molecular crystals, glassy systems, etc.) where the methyl groups are embedded. A collection of harmonic oscillators is usually considered as a tractable model of these phonons. ${ }^{3-5}$

In the case of methyl groups in molecular crystals, a simple model is usually invoked. In this model, the methyl group at very low temperature $(T \approx 1 \mathrm{~K})$ is represented as a rigid rotor in a potential which maintains its rotational symmetry. Usually a threefold potential is taken as a good approximation, yielding the Hamiltonian:

$$
H_{\mathrm{R}}=-\frac{\hbar^{2}}{2 I} \frac{\partial^{2}}{\partial \Phi^{2}}+\frac{V_{3}}{2}(1-\cos 3 \Phi),
$$

with $I$ the moment of inertia and $V_{3}$ the height of the potential barrier. The energy levels, known as librational levels, are tunnel-split into three sublevels labeled by the indexes $A$, $E_{\mathrm{a}}$, and $E_{\mathrm{b}}$, according to the irreducible representations of the symmetry group $C_{3}$ of $H_{\mathrm{R}}$. The levels $E_{\mathrm{a}}$ and $E_{\mathrm{b}}$ have the same energy, yielding the degenerate level $E$, which alternates with $A$ when changing to consecutive librational levels. The energy splitting of the ground librational level is usually referred as the "tunneling frequency," $\omega_{t}$, which, for barriers below $\approx 700 \mathrm{~K}$, can be detected by neutron scattering (NS) in the $\mu \mathrm{eV}$ range as two inelastic peaks for neutron energy gain and loss.,

With increasing temperature, the methyl group is considered coupled to the matrix phonons. Now the Hamiltonian is a sum of three terms: $H=H_{\mathrm{R}}+H_{\mathrm{B}}+H_{\mathrm{C}}$, where $H_{\mathrm{B}}$ is a sum of harmonic oscillator Hamiltonians and $H_{\mathrm{C}}$ is a linear coupling term, which, as $H_{\mathrm{R}}$, is required to be invariant under the $C_{3}$ group. $^{3-5}$ As a consequence of the coupling, the inelastic tunneling spectrum evolves with temperature (see below) to give rise at high temperature (classical limit) to a
PACS number(s): 61.43.Fs, 61.12.Ex, 61.41.+e

single quasielastic spectrum corresponding to classical hopping processes over the potential barrier.

Although this simple approach has been widely applied to methyl group dynamics in different molecular crystals [see, e.g., (Refs. 8-10)], the crossover from rotational tunneling to classical hopping still has not been investigated in systems showing a strong disorder as structural glasses or polymers. The underlying problem is that the simple picture above described breaks down in the case of these systems even in the classical and quantum limits. ${ }^{11-14}$ In the classical limit it produces inconsistent and unphysical results. In the quantum limit, tunneling peaks were expected for a long time but never observed. Only very recently ${ }^{14-16}$ tunneling spectra in several glasses have been directly observed by NS. Instead of well defined tunneling peaks, these spectra show an apparent quasielastic feature even at $1 \mathrm{~K}$. These experimental facts have been rationalized by invoking the simple idea of distributions of potential barriers associated to the structural disorder which is inherent to the glassy state. ${ }^{11-17}$ However, a complete picture of the crossover from quantum to classical dynamics of methyl groups in glasses is still lacking. It is worthy of remark that it is not evident - a priori-whether or not a distribution of potential barriers is the only ingredient needed for a full description of the crossover region, where matrix phonons play an important role. For instance, it is well known that low-frequency phonons in glasses exhibit very different characteristics in comparison with crystals. ${ }^{18}$ On the other hand, it is worth emphasizing that a simple model which were able to explain successfully the crossover from rotational tunneling to relaxation in strongly disordered systems, would become a useful tool: small molecular rotors can be used as internal dynamic probes to obtain information about local disorder and the role of the different interactions in such systems. ${ }^{19}$ In this letter we show that a simple definition for the "crossover temperature" from quantum to classical regime for each methyl group opens a route to extend a previously proposed model for quantum and classical limits to the crossover regime without including any additional parameter. The proposed model is checked by means of NS data of a polymer glass, poly(vinyl acetate) (PVAc), in a wide temperature range from 1 to $200 \mathrm{~K}$.

Within the scenario of our model-rotation rate distribution model (RRDM) - the NS spectra corresponding to methyl group dynamics in a given glass can be understood as the superposition of crystal-like spectra, each one corre- 
sponding to one methyl group in the system. Therefore, at $T \approx 1 \mathrm{~K}$, the NS spectrum of each of these methyl groups will consist of a central elastic peak and two inelastic peaks (Dirac deltas convoluted with the instrumental resolution) centered at $\pm \hbar \omega_{\mathrm{t}}$. As in a crystalline system, the evolution with temperature of each single methyl group spectrum is assumed to be driven by the coupling of the methyl group to a bath of harmonic oscillations. When increasing temperature, the tunneling peaks broaden and shift to the elastic peak and, in addition, a quasielastic broadening appears around the elastic line. These quasielastic and inelastic lines are well described by Lorentzian functions in this temperature range. The two inelastic Lorentzians are due to transitions involving the symmetry change $A \leftrightarrow E$, while the quasielastic Lorentzian involves transitions $E_{\mathrm{a}} \leftrightarrow E_{\mathrm{b}}$. Transitions without symmetry change do not lead to broadening ${ }^{4}$ and remain contributing to the central elastic peak. As in crystalline systems, we will assume that the broadening of these Lorentzian functions with temperature follows Arrhenius laws activated by the first librational energy $E_{01}$ of the methyl group considered,

$$
\Gamma_{A E}=\Gamma_{E_{\mathrm{a}} E_{\mathrm{b}}}=\Gamma_{\mathrm{br}} \exp \left(-E_{01} / k T\right)
$$

for the half-widths-at-half-maximum (HWHM) of the inelastic $\left(\Gamma_{A E}\right)$ and quasielastic $\left(\Gamma_{E_{\mathrm{a}} E_{\mathrm{b}}}\right)$ Lorentzians. In these expressions we have also assumed that the preexponential factor $\Gamma_{\mathrm{br}}$ is the same for both Arrhenius laws. The shift $\left(\hbar \Delta \omega_{\mathrm{t}}\right)$ with temperature of the two inelastic Lorentzians will also follow an Arrhenius law with an activation energy which in crystals is close to $E_{01}$. In our approach this energy is taken just as $E_{01}$. Therefore,

$$
\hbar \Delta \omega_{\mathrm{t}}=\Gamma_{\mathrm{sh}} \exp \left(-E_{01} / k T\right),
$$

where $\Gamma_{\text {sh }}$ is a preexponential factor for the shift. Following the well-known phenomenology of crystalline systems, we will also assume that in a temperature range narrower than $7-10 \mathrm{~K}$, the two inelastic Lorentzians and the quasielastic Lorentzian merge giving rise to a single quasielastic Lorentzian function at higher temperatures. We will refer to this somewhat ill-defined merging temperature as the "crossover temperature," $T_{\mathrm{c}}$, from quantum to classical regime. Above $T_{\mathrm{c}}$ the broadening of the single quasielastic Lorentzian will be driven by the classical activation energy, $E_{\mathrm{A}}$, for methyl group rotation over the potential barrier. Therefore, the HWHM of this Lorentzian function gives the hopping rate

$$
\Gamma=\Gamma_{\infty} \exp \left(-E_{A} / k T\right),
$$

with $\Gamma_{\infty}$ a preexponential factor which in principle could also be different for each methyl group. However, according to previous results, ${ }^{11}$ we will take it as a barrier-independent factor.

In order to define $T_{\mathrm{c}}$ we have considered that the crossover to classical behavior will take place when the incoherent hopping rate becomes comparable to the rate of coherent oscillations. Then $T_{\mathrm{c}}$ is operatively defined by the condition $\Gamma\left(T_{\mathrm{c}}\right) \approx \hbar \omega_{\mathrm{t}}$, which taking into account Eq. (4) reads:

$$
k T_{\mathrm{c}}=E_{A} / \ln \left(\Gamma_{\infty} / \hbar \omega_{\mathrm{t}}\right) .
$$

Taking into account the approximate linear dependence of $E_{A}$ on $V_{3}$ and that of $\ln \left(\hbar \omega_{t}\right)$ on $V_{3}^{1 / 2}$ (Ref. 15), it is easy to see from Eq. (5) that $T_{\mathrm{c}}$ defined in this way also varies approximately with $V_{3}^{1 / 2}$.

It is worth emphasizing that in the framework of this approach, the existence of a distribution of potential barriers $V_{3}$ for methyl group dynamics in glassy systems implies not only a distribution of $\hbar \omega_{\mathrm{t}}, E_{A}$, and $E_{01}$, but also a distribution of crossover temperatures $T_{\mathrm{c}}$, which can be directly calculated from the distribution of $V_{3}$, once the relationship between $T_{\mathrm{c}}$ and $V_{3}$ is known.

In an ideal crystalline system the shift and broadening of the tunneling peaks can be experimentally observed. Thereby, the preexponential factors $\Gamma_{\mathrm{br}}$ and $\Gamma_{\mathrm{sh}}$ can be experimentally determined in a model-independent way. This is not the case in structural glasses, where individual peaks are not visible. In our model, these preexponential factors can be eliminated taking into account our definition of $T_{\mathrm{c}}$. This can be done by imposing at $T_{\mathrm{c}}$ the continuity condition $\Gamma_{A E}\left(T_{\mathrm{c}}\right)=\Gamma_{E_{\mathrm{a}} E_{\mathrm{b}}}\left(T_{\mathrm{c}}\right)=\Gamma\left(T_{\mathrm{c}}\right)$, and taking into account that at the crossover temperature, which marks the onset of the classical behavior, the tunneling frequency will be shifted to zero energy: $\hbar \Delta \omega_{\mathrm{t}}\left(T_{\mathrm{c}}\right)=\hbar \omega_{\mathrm{t}}$. Since $\hbar \omega_{\mathrm{t}}, E_{01}$, and $E_{A}$ are directly obtained as functions of $V_{3}$ by solving the Schrödinger equation for $H_{R}$, we have that, if the values of $V_{3}$ and $\Gamma_{\infty}$ are given, $\Gamma_{\mathrm{br}}$ and $\Gamma_{\mathrm{sh}}$ can be obtained for each methyl group.

Therefore, with Eqs. (2)-(5) and the two latter conditions, the behavior of the individual spectra of each methyl group is unambiguously determined by the values of $V_{3}$ and $\Gamma_{\infty}$, these two parameters controlling when the methyl group reaches the classical behavior, as it is indicated by Eq. (5). The preexponential factor $\Gamma_{\infty}$ can be seen as a measure of the strength of the coupling of the methyl group to the matrix phonons. For high values of $\Gamma_{\infty}$, i.e., for a strong coupling, the classical behavior will be reached at lower temperatures.

Now we will present how to adopt the above described model to the NS functions. The incoherent scattering function in the low-temperature limit (tunneling regime) is given by: $:$,7

$$
\begin{aligned}
S_{\mathrm{MG}}^{\mathrm{inc}}(Q, \omega)= & \frac{5+4 j_{0}(Q r)}{9} \delta(\omega)+\frac{2\left[1-j_{0}(Q r)\right]}{9} \\
& \times\left[\delta\left(\omega+\omega_{\mathrm{t}}\right)+\delta\left(\omega-\omega_{\mathrm{t}}\right)\right]
\end{aligned}
$$

where the factors $\left[1+2 j_{0}(Q r)\right] / 3$ and $2\left[1-j_{0}(Q r)\right] / 9$ correspond, respectively, to transitions without and with symmetry change. ${ }^{6} j_{0}$ is the zeroth-order Bessel function and $r$ the $\mathrm{H}-\mathrm{H}$ distance in the methyl group. As we mentioned above, when increasing the temperature, the Dirac deltas for the transitions with symmetry change are substituted by Lorentzians, while the Dirac delta for transitions without symmetry change is retained, so for the crossover regime:

$$
\begin{aligned}
S_{\mathrm{MG}}^{\mathrm{inc}}(Q, \omega)= & \frac{1+2 j_{0}(Q r)}{3} \delta(\omega)+\frac{2\left[1-j_{0}(Q r)\right]}{9} \\
& \times\left\{L\left(\omega ; \Gamma_{E_{\mathrm{a}} E_{\mathrm{b}}}\right)+L\left[\omega+\left(\omega_{\mathrm{t}}-\Delta \omega_{\mathrm{t}}\right) ; \Gamma_{A E}\right]\right. \\
& \left.+L\left[\omega-\left(\omega_{\mathrm{t}}-\Delta \omega_{\mathrm{t}}\right) ; \Gamma_{A E}\right]\right\} .
\end{aligned}
$$


The intensities of the lines are the same as in the lowtemperature limit, since they only depend on the symmetry group $C_{n}$ of the Hamiltonian, ${ }^{6}$ which is the same $(n=3)$ for $H_{\mathrm{R}}$ and $H$.

At high-temperatures a classical picture of three particles interchanging their positions emerges. If the residence time between hoppings (assumed to be instantaneous) is $\tau$ $=3 / 2 \Gamma$, then we have: 6

$$
S_{\mathrm{MG}}^{\mathrm{inc}}(Q, \omega)=\frac{1+2 j_{0}(Q r)}{3} \delta(\omega)+\frac{2\left[1-j_{0}(Q r)\right]}{3} L(\omega ; \Gamma) \text {. }
$$

It is straightforward to see that the crossover function (7) reduces to Eqs. (6) and (8) in the two temperature limits: at very low temperature the three Lorentzians have zero width and the inelastic ones are not shifted, recovering Eq. (6). In the classical limit $\Delta \omega_{\mathrm{t}}=\omega_{\mathrm{t}}$, yielding Eq. (8) if $\Gamma_{A E}$ and $\Gamma_{E_{\mathrm{a}} E_{\mathrm{b}}}$ are now substituted by the classical $\Gamma$.

With the definition of the crossover temperature given above, the incoherent scattering function $S_{\mathrm{MG}}^{\mathrm{inc}}(Q, \omega)$ for a given methyl group in the system will be taken as Eq. (7) for $T<T_{\mathrm{c}}$ and as Eq. (8) for $T>T_{\mathrm{c}}$ with the quantities $T_{\mathrm{c}}, \Delta \omega_{\mathrm{t}}$, $\Gamma_{A E}, \Gamma_{E_{\mathrm{a}} E_{\mathrm{b}}}$, and $\Gamma$ determined by Eqs. (2)-(5). We would like to emphasize that, according to this approach, all the quantities appearing in these equations and therefore, also the scattering functions, depend exclusively on $V_{3}$ and $\Gamma_{\infty}$. Thus, in the framework of the RRDM, the incoherent scattering function will be given by the superposition of the individual scattering functions defined above, weighted by the distribution of potential barriers $g\left(V_{3}\right)$ :

$$
S_{\mathrm{RRDM}}^{\mathrm{inc}}(Q, \omega)=\int g\left(V_{3}\right) S_{\mathrm{MG}}^{\mathrm{inc}}\left(Q, \omega, V_{3}\right) d V_{3} .
$$

As in many situations involving disorder [see, e.g., (Ref. 20)], $g\left(V_{3}\right)$ is assumed to be a Gaussian function:

$$
g\left(V_{3}\right)=\left(1 / \sqrt{2 \pi} \sigma_{\mathrm{V}}\right) \exp \left[-\left(V_{3}-V_{3_{0}}\right)^{2} / 2 \sigma_{\mathrm{V}}^{2}\right]
$$

For comparison with the experimental spectra, the total coherent contribution and the incoherent scattering of the other atoms in the sample, which are assumed to be fixed, are added to obtain the total scattering function $S(Q, \omega) \cdot{ }^{15,16} \mathrm{Fi}$ nally, $S(Q, \omega)$ has to be convoluted with the instrumental resolution function $R(Q, \omega)$.

Recent NS measurements on PVAc, carried out in the high-resolution backscattering spectrometer IN16 of the ILL (Grenoble, France), had been successfully interpreted in terms of the RRDM in the tunneling and classical limits, ${ }^{14,17}$ the latter observed above $\approx 70 \mathrm{~K}$. The parameters of the model were found to be $\Gamma_{\infty}=9.1 \mathrm{meV}, V_{3_{0}}=534 \mathrm{~K}$, and $\sigma_{\mathrm{V}}=274 \mathrm{~K}^{21}$ Once these parameters are known, the extended RRDM described above should give account for the experimental spectra in the whole temperature range. This is illustrated in Fig. 1, which shows spectra corresponding to the three regimes: tunneling $(2 \mathrm{~K})$, crossover $(30 \mathrm{~K})$ and classical $(70 \mathrm{~K})$ in excellent agreement with the theoretical spectra given by the RRDM.

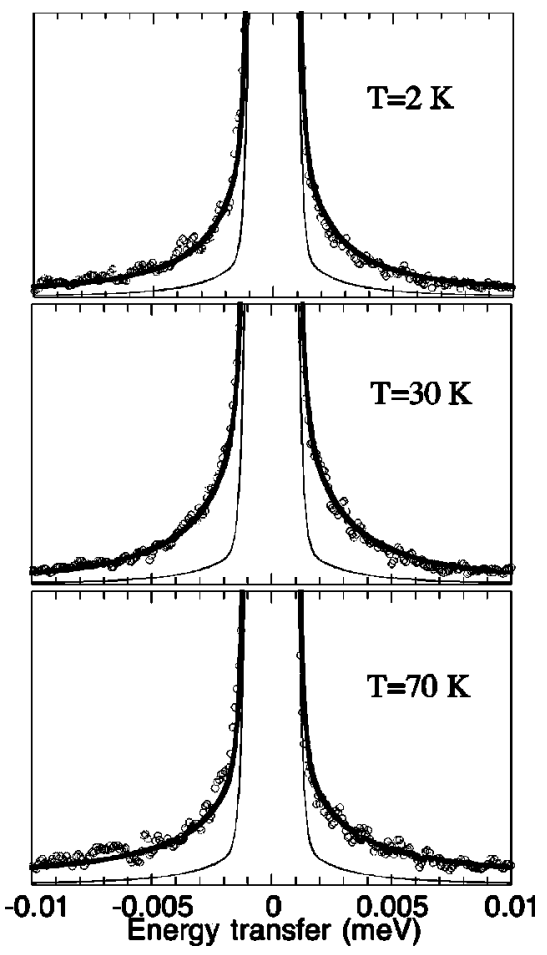

FIG. 1. Neutron-scattering spectra for PVAc at $Q=1.75 \AA^{-1}$ and different temperatures. Scale: $5 \%$ of the maximum. Circles are the experimental points. Thick lines are the theoretical spectra according to the RRDM. Thin lines correspond to the resolution function.

A better way to follow in detail the evolution of the spectra with temperature can be obtained depicting inelastic integrated intensities vs temperature. This is shown in Fig. 2 for three different inelastic windows covering the dynamic range of the IN16 spectrometer. As can be seen, the evolution of the inelastic integrated intensities with temperature reveals a double-peak structure which was not visible in Fig. 1. It is worth emphasizing that this structure is not due to any experimental artifact. It should also be observable in crystalline systems when the inelastic window is properly chosen, e.g., close, but at lower energies, to the tunneling peak at low temperature. With increasing temperature, the tunneling peak

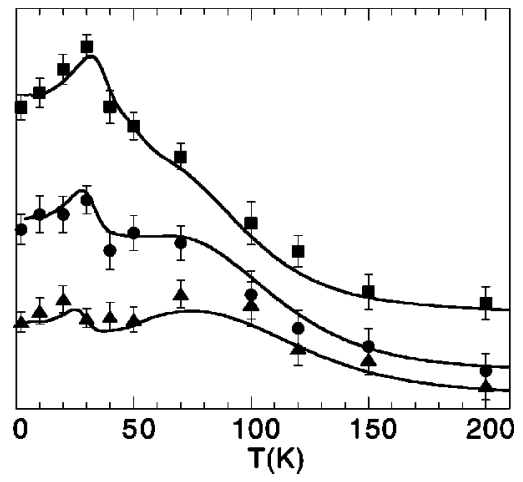

FIG. 2. Integrated intensities at $Q=1.75 \AA^{-1}$ in the ranges $1-2.5 \mu \mathrm{eV}$ (squares), 2.5-6 $\mu \mathrm{eV}$ (circles), and 6-10 $\mu \mathrm{eV}$ (triangles). The lines are the values predicted by the RRDM. 
starts to broaden and moves towards lower energies. As a consequence, the intensity in the inelastic window will pass through a first maximum. A second maximum will be visible at higher temperatures when the broadening of the quasielastic line reaches the inelastic window. In glasses, due to the broad distribution of tunneling lines in comparison with the maximum dynamic range of the spectrometer, we should expect to observe a double peak structure at any inelastic window, though with different relative intensities of the peaks. Figure 2 also includes the curves deduced from the parameters of the RRDM and corrected by a Debye-Waller factor as described elsewhere. ${ }^{14}$ The agreement of the theoretical curves with the experimental points is excellent, nicely reproducing the double peak structure commented above.

In summary, we have shown that a model based on the simple idea of superposition of crystal-like states can nicelyexplain all the features involved in a complicated problem: the crossover from coherent rotational tunneling to relaxation of methyl groups in polymer glasses. In this approach, the parameter $\Gamma_{\infty}$ carries the information about the coupling between the molecular rotors and the matrix phonons. Thus, different values of $\Gamma_{\infty}$ should be expected for a glassy system and its crystalline counterpart. Unfortunately, this comparison cannot be carried out in polymers where the crystalline state is usually not well defined. However, it can be done in the case of low molecular weight glasses as, e.g., sodium acetate trihydrate, where both glassy and crystalline states exist. ${ }^{16}$ Work in this direction is in progress. On the other hand, since the model here proposed is quite general, it can also be applied to the problem of the crossover in systems other than glasses or polymers but where disorder plays an important role. One interesting possibility would be the dynamics of small molecular rotors in confined geometries.

This work has been supported by the Spanish Ministry of Education (project PB97-0638), the Government of the Basque Country (projects GV-PI98/20 and EX-1999/11), and the University of the Basque Country (project 206.215-G20/ 98). The support of the Donostia International Physics Center and Iberdrola S.A. is also acknowledged. A.J.M. acknowledges a grant of the Basque Government. We also thank Dr. B. Frick for experimental support.
${ }^{1}$ W.H. Zurek, Phys. Today 44 (10), 36 (1991).

${ }^{2}$ S. Clough, A. Heidemann, A.J. Horsewill, J.D. Lewis, and M.N.J. Paley, J. Phys. C 15, 2495 (1982).

${ }^{3}$ A.C. Hewson, J. Phys. C 15, 3841 (1982).

${ }^{4}$ A. Würger, Z. Phys. B: Condens. Matter 76, 65 (1989).

${ }^{5}$ A. Hüller, Z. Phys. B: Condens. Matter 78, 125 (1990).

${ }^{6}$ W. Press, in Single-Particle Rotations in Molecular Crystals, Vol. 92 of Springer Tracts in Modern Physics (Springer, Berlin, 1981).

${ }^{7}$ M. Prager and A. Heidemann, Chem. Rev. 97, 2933 (1997).

${ }^{8}$ D. Cavagnat, A. Magerl, C. Vettier, and S. Clough, J. Phys. C 19, 6665 (1986).

${ }^{9}$ A. Heidemann, M. Prager, and M. Monkenbusch, Z. Phys. B: Condens. Matter 76, 77 (1989).

${ }^{10}$ M. Prager, J. Stanislawski, and W. Häusler, J. Chem. Phys. 86, 2563 (1987).

${ }^{11}$ A. Chahid, A. Alegría, and J. Colmenero, Macromolecules 27, 3282 (1994).

${ }^{12}$ B. Frick and L.J. Fetters, Macromolecules 27, 974 (1994).
${ }^{13}$ V. Arrighi and J.S. Higgins, Physica B 226, 1 (1996).

${ }^{14}$ J. Colmenero, R. Mukhopadhyay, A. Alegría, and B. Frick, Phys. Rev. Lett. 80, 2350 (1998).

${ }^{15}$ A.J. Moreno, A. Alegría, J. Colmenero, and B. Frick, Phys. Rev. B 59, 5983 (1999).

${ }^{16}$ A.J. Moreno, A. Alegría, J. Colmenero, and B. Frick, Physica B 276-278, 361 (2000).

${ }^{17}$ R. Mukhopadhyay, A. Alegría, J. Colmenero, and B. Frick, Macromolecules 31, 3985 (1998).

${ }^{18}$ G.P. Srivastava, The Physics of Phonons (Adam Hilger, Bristol, 1990).

${ }^{19}$ F. Alvarez, A. Alegría, J. Colmenero, T.M. Nicholson, and G.R. Davies, Macromolecules 33, 8077 (2000).

${ }^{20}$ C. Bostoen, G. Coddens, and W. Wegener, J. Chem. Phys. 91, 6337 (1989).

${ }^{21}$ It is noteworthy that the maximum value of the corresponding distribution of $E_{01}(15.7 \mathrm{meV})$ agrees quite well with the experimental value $(\approx 15 \mathrm{meV})$ measured by NS in the spectrometer TOSCA at ISIS (Chilton, U.K.) (Exp. Rep. No. 9638). 